\title{
ACUTE LOSS OF VISION IN A YOUNG WOMAN: A CASE REPORT ON PSYCHOGENIC BLINDNESS
}

\author{
Mulugeta $S^{1}$, Tesfay $K^{1}$, Frank $R^{2}$, Gruber-Frank $C^{2}$
}

\begin{abstract}
BACKGROUND: Acute loss of vision needs urgent attention and treatment. We report on a young Ethiopian woman who experienced acute bilateral blindness. In the presence of normal ophthalmological findings psychogenic blindness has to be considered.

CASE DETAILS: A 21 years old woman was admitted to the psychiatry clinic at Jimma University specialized Hospital, Jimma, South West Ethiopia. She had not been able to see for a few days. Ophthalmological and neurological examinations showed normal findings. No severe psychopathology was found. Stressful life events had preceded the appearance of the symptoms. An attitude of accepting the perspective of the patient of a seemingly organic disease is a precondition for a successful intervention. Treatment aimed at a gradual return to normal functioning. The patient regained her sight and fully recovered. The final psychiatric diagnosis was psychogenic blindness.

CONCLUSION: The psychosocial intervention was successful and encouraging for the patient and clinicians.

KEYWORDS: acute loss of vision, psychosocial intervention, conversion disorder, low-income country, international cooperation.
\end{abstract}

DOI: http://dx.doi.org/10.4314/ejhs.v25i1.13

\section{INTRODUCTION}

The most frequent cause of visual impairment in about $50 \%$ of all forms of blindness in all areas of the world, except for developed countries is cataract. In the least-developed countries, particularly in Sub-Saharan Africa, the causes of avoidable blindness are primarily cataract $(50 \%)$, glaucoma (15\%), corneal opacities (10\%), trachoma $(6.8 \%)$, childhood blindness $(5.3 \%)$ and onchocerciasis (4\%) (1). Visual function is defined in four levels: normal, moderate visual impairment, severe visual impairment and blindness. The most frequent causes of visual impairment are uncorrected refractive disorders, unoperated cataract and glaucoma (2). Persons with visual impairment as well as persons with blindness need to adapt to everyday demands.
Acute loss of vision needs urgent attention and treatment. Acute visual loss may be due to trauma or infection. Transitory impairment of vision can be due to epilepsy or migraine (3). Loss of vision despite normal ophthalmological findings, accompanied by neurological abnormalities, indicates that the visual cortex of the brain is affected.

Acute loss of vision as well as loss of voice may have an organic and a psychogenic etiology. The majority of people affected by the conditions seek help in health care facilities with an organic orientation. When examinations result in normal findings, patients are sent towards a mental health care facility. The symptoms are summarized under the heading of "emotional and medically unexplained medical complaints" and are a priority condition in mental health (4). The national

\footnotetext{
${ }^{1}$ Department of Psychiatry, Jimma University, Ethiopia

${ }^{2}$ Global Mental Health Group, Center International Health, Ludwig Maximilians University, Munch Germany

Corresponding Author: Mulugeta S, sisaym8@gmail.com
} 
mental health strategy of Ethiopia identified limited resources for non-medication alternatives for rehabilitation, for psychological treatments and for children as weaknesses of the mental health system (5).

We report on a young woman who experienced acute bilateral blindness preceded by an episode of loss of voice (aphonia). The purpose of this case report is to describe the implementation of a psychosocial intervention in a health system with limited resources for mental health care (6). Informed consent for the publication was obtained from the patient.

\section{CASE DETAILS}

The 21 years old female patient came to Jimma University Specialized Hospital accompanied by her mother. The chief complaint of the patient was: "I haven't been able to see for seven days". She was a $3^{\text {rd }}$ year university student at another Ethiopian university. She was single and never married, protestant by religion and Amhara by ethnicity, and she was living in Jimma.

At the ophthalmic clinic, an ophthalmologic evaluation was done. Visual acuity was diminished to +3 dioptres on both eyes, and had already been corrected by glasses. All other visual functions were normal. Malingering was suspected and, a psychiatric consultation was made. At the initial contact at the Department of Psychiatry, the patient was alert and conscious. She was also fully oriented to time, place and person. She had good immediate, recent and remote memory. Concentration and attention were normal. The patient had good social and test judgment. She was smiling and reported to feel nothing despite acute severe visual impairment. No severe psychopathological abnormalities could be elicited. Vital signs were within normal range.

An approach to the patient was developed through cooperation among the local Ethiopian clinicians and guest lecturers, a child psychiatrist and a children's nurse, who took part in an international exchange program (7).

A neurological examination was performed. The patient was cooperative. She could perfectly understand all tasks necessary to do a full neurological examination.

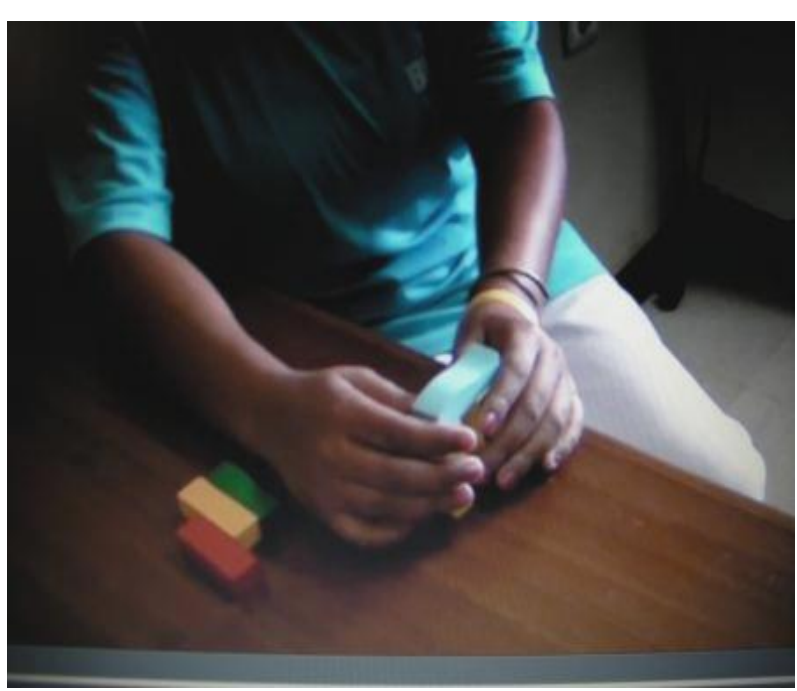

Figure 1: Reproducing shape of blocks

The patient was able to reproduce the set-up of blocks of wood. The series of patterns presented with an increasing difficulty. She indicated a narrow concentric visual field. She was slow and very careful when performing tasks. The examiner's feedback to the patient was that she had good abilities. However, she remained very cautious and was performing slowly. When encouraged after some exercises, she performed faster. Overall, the neurological examination was normal.

This was her second visit to the psychiatric clinic. A month before her visit to the clinic, she had had difficulties in studying for her BSc degree. At the same time, her mother informed her about a life threatening illness of her father. A few hours later, she became unable to speak. This inability to speak lasted for two weeks. One of her friends helped her to train to speak and she recovered. No treatment in any health care facility was necessary. One week later, the patient was preparing for an exam to be held the next day. In the early evening, she became completely unable to see. Due to the impairment of her vision, the patient was not able to sit for her exam. She reported feeling of occasional sadness, crying, hopelessness and suicidal idea but without plan or attempt.

Eight months prior to her presentation, she became voiceless for about one and half months, and was admitted to the psychiatric clinic. She was examined then by laryngoscopy and found to be normal. She was treated with $5 \mathrm{mg}$ diazepam at bed time, and some psychotherapy made her free 
of stress and anxiety like imaginary relaxation technique, slow breathing and progressive muscle relaxation therapy. After 3 days of therapy, she was able to produce sounds like coughing. On the fourth day, she was able to say a few words and the next day she became fully communicative.

Her development was normal. At the age of 10 , about 10 years ago, she had suffered a car accident-she had been unconscious for 10 days. After awakening from unconsciousness, her left side was paralyzed and she was not able to hear with her left ear. Later, she regained her muscle tone. Actually, her left side was still slightly weaker when compared to her right side. She had discontinued school for 1 year because of the head trauma. She was a middle ranking student at school.

She complained of almost daily headache. She had occasional falling attacks characterized by loss of consciousness but no jerky movements and no muscle rigidity. They started few years after the trauma and are still persisting occasionally. The patient did not get any anti-epileptic medication. There were no psychiatric problems in the family.

The diagnostic formulation according to the psychiatric classification system DSM IV-TR relies on the history and the clinical findings: "Conversion disorder with mixed presentation (sensory =vision and motor = voice)". Other health problems were migraine headache, and worries about seizures (8).

Most important for the management, seems having knowledge of this condition. The basic element is an attitude to accept the patient's views and to build bridges. There is no standardized treatment approach to psychogenic blindness. A treatment program was tailored to the situation of the clinic and to the needs of the patients mainly relying on the WHO mental health GAP intervention guide, section "significant emotional or medically unexplained complaints" (4). The approach was symptom oriented on how to give orientation to a blind person irrespective of etiology.

The children's nurse offered material suitable for a blind person to the patient. The patient was asked to reproduce the pattern of different shapes by means of little knobs which had to be placed into a plate with holes.

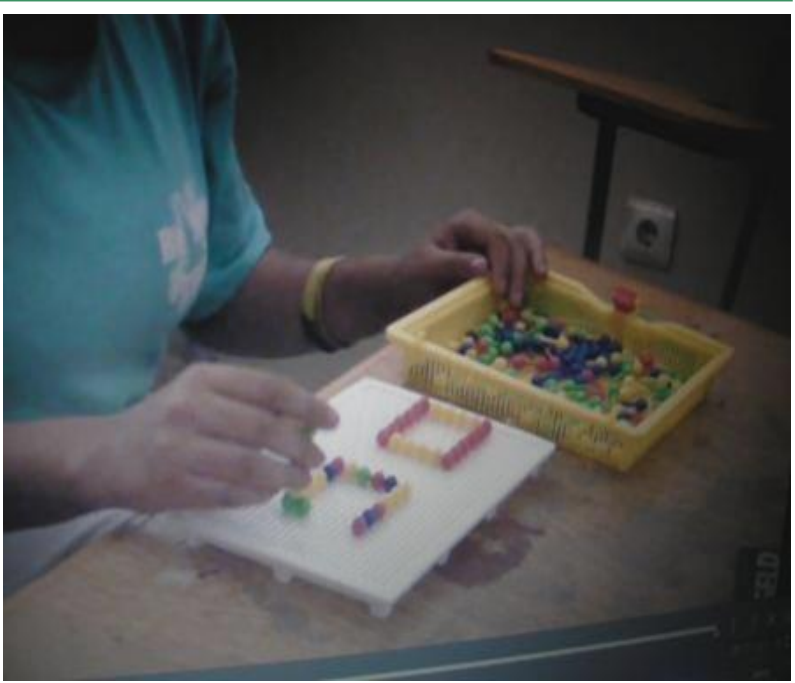

Figure 2: Reproducing shape of knobs

An attempt was made to offer painting material to her as to other patients. She was able to draw nice scenery using different colors and to paint precisely a face (Figure 3 ). Later on, she was able to make pearls from paper by wrapping colored paper around a stick of wood. It was proved that she was faster than all other patients. She could make a necklace for herself and she provided a lot of paper pearls to other patients.

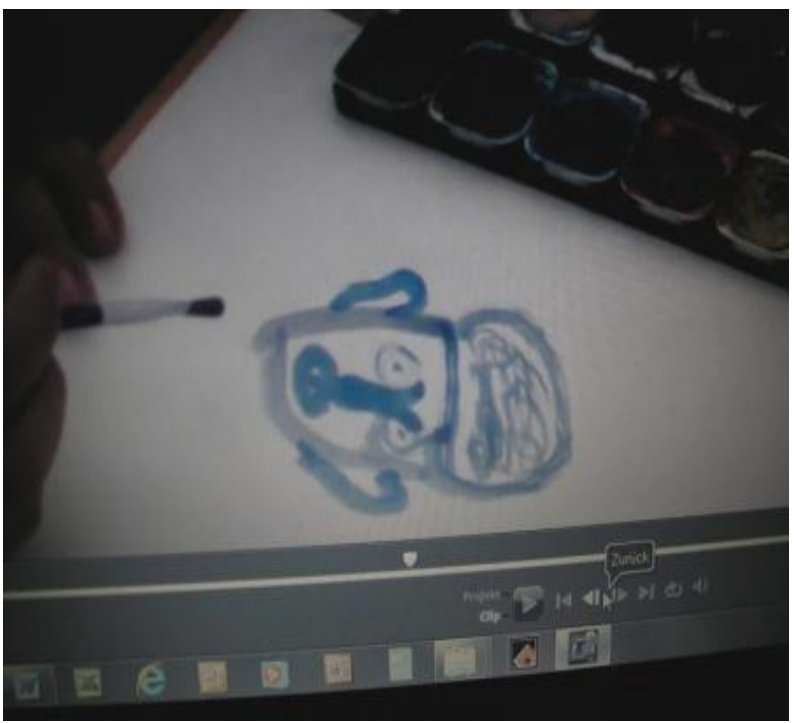

Figure 3: Painting skills 


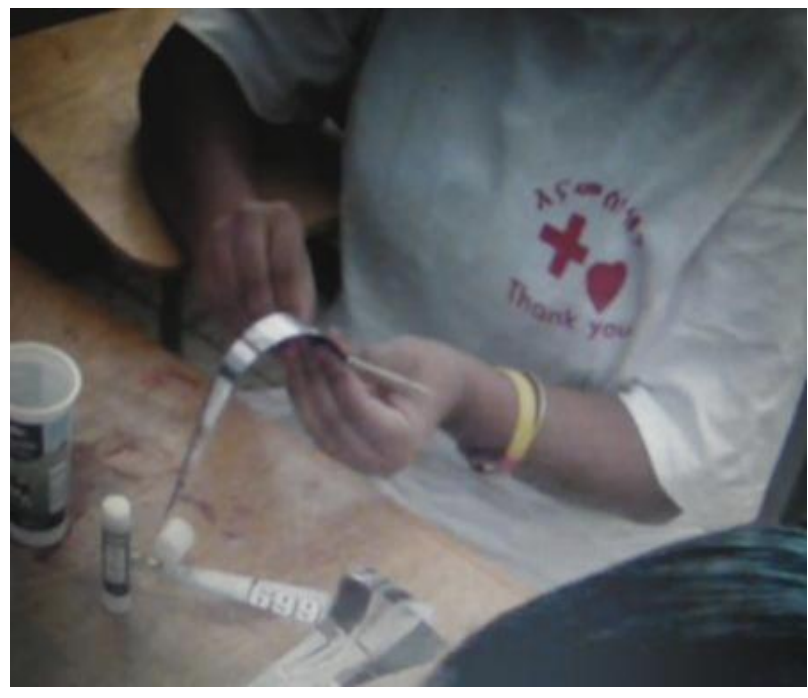

Figure 4: Making paper pearls

The patient presented herself as an insecure person. She said that she could not see what she was doing. She frequently controlled her performance and was finally successful. There was a discrepancy between her attitude of an insecure, dependent blind person while reproducing shapes of blocks and the elaborate drawing she was able to do. Her performance corresponded to a narrow visual field seen during the neurological examination. Nurses and clinicians encouraged the patient and praised her for her skills and abilities. She seemed unable to believe the compliments given to her.

In addition, she received imaginary relaxation technique, slow breathing and progressive muscle relaxation therapy by SM. Diazepam $10 \mathrm{mg}$ orally was given daily for 1 week and $5 \mathrm{mg}$ for another1 week, then stopped. Amitriptyline $25 \mathrm{mg}$ daily for her migraine and occasional depressive symptoms.

The patient started to improve after 1 week. Initially, she started to see slight flash light. She became opener in contact, and she began to recognize some people, beginning with her mother, who accompanied her. She participated in group work: put leaves on a paper to form a tree.

She was discharged from the hospital after 3 weeks, when she was able to see normally. At first follow up as an outpatient after two days, she showed good and adequately modulated mood, bright and could see normally. She is on further follow up. Table 1 summarizes key elements of the intervention.

Table 1: Key elements of the intervention (Adapted from reference 4)

\section{Basic interventions -General principles of care}

Build up a supportive relationship. - Accept the patient's view. Build bridges.

Provide information on the health status in terms the patient can understand.

Communicate realistic hope for better functioning and recovery.

Continually monitor for treatment effects and outcome.

Offer occupation for a person with vision problems:

Touch and feel blocks, knobs, paper pearls

Give constructive feedback

At follow up reassess the persons understanding of treatment.

Make efforts to link the person to community support.

\begin{tabular}{c}
\hline Advanced interventions \\
\hline Behavioral activation \\
Social skill therapy
\end{tabular}

\section{DISCUSSION}

Psychogenic blindness may be suspected when patients complain of recent onset of blindness without injury. At a closer look, frequently, there may be a diminution of vision, blurred vision or a "tunnel vision" (9). In psychiatric terms, this condition is a type of conversion disorder. The common feature of the whole group of disorders is the prominence of somatic symptoms associated with significant distress and impairment (8).

Due to the many different languages spoken in Ethiopia a lot of descriptions and expressions indicating somatic symptoms resulting from distress appear difficult to translate into English. Only some terms can be translated into psychiatric terminology. Students before or during examinations complained of "heat in the 
head" (10). Ethiopians may use the word "makatel" "burning" or complain of "yazorehnyal", "spinning,". In medical terms, it means vertigo, but it seems to have a broader meaning. From the view of clinicians, the symptoms may seem diffuse, exaggerated and difficult to pinpoint (11).

Conversion disorders are characterized by signs and symptoms clearly inconsistent with what is known about anatomy and pathophysiology (8, 9). Symptoms are exacerbating after stressful events (8). Patients who suffer from conversion symptoms do not intentionally fabricate such symptoms, as malingerers do. The diagnosis of conversion disorder should be made after appropriate investigations that can exclude neurologic conditions, medical conditions, and other psychological disorders. However, organic and psychogenic conditions such as epileptic seizures and psychogenic seizures can co-occur in one person.

Individuals with prominent somatic symptoms are commonly encountered in primary care and other medical settings, but are less commonly encountered in psychiatric and other mental health settings (8). Conversion disorders are two to three times more common in females. There are little data on the prevalence of conversion disorder. Estimates have to rely on the results of clinical investigations.

In Gonder, North West Ethiopia, 1240 patients were seen as first attendees in the outpatient department within half a year in 1986. One hundred and fifty-five or $12.5 \%$ were classified as disorders from hysteric type or conversion disorders in the actual classification. Motor disturbances and seizures were the symptoms most frequently seen. Sixteen patients (1\%) had sensory symptoms and another 16 had multiple symptoms. These behavior patterns were interpreted as mechanisms of problem-solutions with secondary gain of illness under rapidly changing social conditions (12). There was a large group of military men with motor disturbances unable to continue the service and a small group of students who needed permission to repeat a year of study or to change fieldof study. Initial episodes of conversion symptoms remit spontaneously, often within weeks or months. Complications perpetuating the duration of illness arise when patients restrict their activities such as staying in bed. Jobs may be lost and relationships strained. Physician-initiated perpetuating factors are potentially dangerous or costly diagnostic procedures and interventions (8).

Looking at the history of our patient and reflecting on precipitating and perpetuating factors in previous experiences helps to predict possible relapses when emotionally difficult situations reoccur (13). A major predisposing factor is the severe head trauma at age 10. Being unconscious for ten days and having motor problems and hearing problems for a long time indicates a severe affection of the brain with slow recovery. Normal functioning at school was possible only one year after the accident. Headaches were frequent afterwards. During the actual hospitalization, the neurological examination was normal as well as the performance of some tasks testing cognitive functioning. However, it was not possible to do an in depth examination of cognitive abilities. Functioning in everyday situations can be normal. Problems may arise due to minor cognitive impairment in situations with high demandseither emotional or cognitive -and result in a breakdown of abilities. The patient had experienced emotionally stressful situations: A life threatening illness of a close family member accompanying the demands of a final examination can be interpreted as precipitating event. No perpetuating factors were found.

There are several protective factors. For both hospitalizations, the access to the clinic was short and direct. No unnecessary diagnostic or therapeutic measures had been undertaken. The family and friends were very supportive. Previously, the patient had experienced training as helpful. The intention of the training was to build a bridge for the patient by accepting her inability to see. From detailed observation of the patient in different situations, a plan of management could be developed. Training of visual functions was accompanied by behavior activation and social skills therapy.

The WHO mhGAP intervention guide (4) excludes psychotropic medications for the management of "medically unexplained and emotional symptoms". A meta-analysis done on the evidence drug effectiveness showed that 
effectiveness of drugs is of poor quality, but may be of use in the treatment of acute and treatmentresistant conversion disorder (14). Patients may benefit from psychotherapy. Psychotherapeutic treatments have to be targeted in a range of potentially modifiable factors such as illness perceptions and emotionally processing of previous traumatic events (15). In the United Kingdom, patients with psychogenic seizures had been treated by short psychotherapy. At follow up after about 4 years, $25 \%$ became seizure free and $40 \%$ had seizure reduction. The only predictor of seizure cessation from baseline data was "economic activity". Health care utilization had declined significantly from baseline to follow-up (16). On the long term, the patient should be supported to recognize difficult situations in time such as how to prepare an examination and to learn how to handle them.

In conclusion, a complicated situation could be managed successfully in a specialized mental health facility by local means and external support. The reflection on the process and the outcome is encouraging. As suggested by the National Mental Health Strategy (5), supervision and ongoing training of clinicians and the nursing staff should be implemented to be prepared for patients in similar situations.

\section{REFERENCES}

1. World Health Organization (WHO), Global data on visual impairment, Prevention of Blindness and Visual Impairment, 2010

(http://www.who.int/blindness/causes/en/index.ht $\mathrm{ml}$ (accessed 1410 2013).

2. World Health Organization (WHO), Fact Sheet $\mathrm{N}^{\circ} 282$ Visual impairment and blindness Updated October 2013 www.who.int/mediacentrelfactsheets/fs282/en/ accessed $6 / 5 / 2014$

3. Doummar D, Roussat B, Pelosse B. Management of acute visual loss in children. Arch Pediatr. 2004;11:1384-8.

4. World Health Organization (WHO), mhGAP intervention guide (mental health GAP) 2010. http://www.who.int/mental_health/mhgap/evidenc e/child/en/index.html

5. National Mental Health Strategy 2012: Ethiopia launches its National Mental Health Strategy. http://www.centreforglobalmentalhealth.org/news -events/news/ethiopia-launches-itsnationalmental-health-strategy (accessed 29/3 /2013).
6. Gagnier JJ, Riley D, Altman DG, Moher D, Sox H, Kienle G. 2013. The CARE guidelines. Consensus-based clinical case reporting guideline development. Dtsch Arztebl Int, 110;37:603-8. doi: 10.3238/arztebl.2013.0603

7. Tesfaye M, Abera M, Gruber-Frank C, Frank R. The development of a model of training in child psychiatry for non-physician clinicians in Ethiopia. Child and Adolescent Psychiatry and Mental Health, 2014. http://www.capmh.com/content/8/1/6.

8. American Psychiatric Association Task Force on DSM-IV. Diagnostic and Statistical Manual of Mental Disorders, 4th ed (text revision). Washington, DC: American Psychiatric Association; 2000. American psychiatric association,

Psych.org/MainMenu/Research/DSMIV.aspx.

9. ICD-10 (International classification of diseases, tenth revision): Classification of Mental and Behavioral Disorders: Clinical Descriptions and Diagnostic: World Health Organization: Geneva: 1993

www.who.int/classifications/icd/en/index.html.

10. Petros S, Schier E. Traditional attitudes towards mental illness in Ethiopia. Curare, 1989;12:161167.

11. Giel R, GezahegnY, van LuijkJN. Psychiatric morbidity in 200 Ethiopian medical outpatients. PsychiatrNeurol Neurochir, 1968; 71:169-176.

12. Schier E, Yecunnoamlack $T$, Tegegne $T$. Hysterical syndromes in a population of ambulatory neuropsychiatric patients in northwest Ethiopia. Phenomenology, psychosocial and sociocultural background. Psychiatr Neurol Med Psychol, 1989, 41:161-9.

13. Reuber M, Howlett S, Khan A, et al. Nonepileptic seizures and other functional neurological symptoms: predisposing, precipitating, and perpetuating factors. Psychosomatics, 2007; 48:230e8.

14. Poole NA, Wuerz A, Agrawal N. Abreaction for conversion disorder: systematic review with metaanalysis. British Journal of Psychiatry, 2010;197:91-95.

15. Howlett S, Reuber M. An augmented model of brief psychodynamic interpersonal therapy for patients with nonepileptic seizures. Psychotherapy, 2009; 46:125e38.

16. Mayor R, Howlett S, Gruenewald R, Reuber M. Long-term outcome of brief augmented psychodynamic interpersonal therapy for psychogenic nonepileptic seizures: Seizure control and health care utilization. Epilepsia, 2010;51:1169-1176,.doi: $\quad 10.1111 / j .1528$ 1167.2010.02656.x. 